\title{
Tyramine 1 Receptor Distribution in the Brain of Corbiculate Bees Points to a Conserved Function
}

\author{
Markus Thamm ${ }^{\mathrm{a}}$ Katharina Wagler ${ }^{\mathrm{a}}$ Axel Brockmann ${ }^{\mathrm{b}}$ Ricarda Scheiner ${ }^{\mathrm{a}}$ \\ a Behavioral Physiology and Sociobiology, Julius Maximilian University of Würzburg, Würzburg, Germany; \\ ${ }^{b}$ National Centre for Biological Sciences, Tata Institute of Fundamental Research, Bangalore, India
}

\section{Keywords}

Eusociality · Corbiculate bees · Tyramine $\cdot$ Receptor expression · Gustatory responsiveness

\begin{abstract}
Sucrose represents an important carbohydrate source for most bee species. In the Western honeybee (Apis mellifera) it was shown that individual sucrose responsiveness correlates with the task performed in the colony, supporting the response threshold theory which states that individuals with the lowest threshold for a task-associated stimuli will perform the associated task. Tyramine was shown to modulate sucrose responsiveness, most likely via the tyramine 1 receptor. This receptor is located in brain areas important for the processing of gustatory stimuli. We asked whether the spatial expression pattern of the tyramine 1 receptor is a unique adaptation of honeybees or if its expression represents a conserved trait. Using a specific tyramine receptor 1 antibody, we compared the spatial expression of this receptor in the brain of different corbiculate bee species, including eusocial honeybees, bumblebees, stingless bees, and the solitary bee Osmia bicornis as an outgroup. We found a similar
\end{abstract}

karger@karger.com www.karger.com/bbe

Karger $\stackrel{\text { ' }}{5}$

BOPEN ACCESS
(C) 2021 The Author(s)

Published by S. Karger AG, Basel

This is an Open Access article licensed under the Creative Commons Attribution-NonCommercial-4.0 International License (CC BY-NC) (http://www.karger.com/Services/OpenAccessLicense), applicable to the online version of the article only. Usage and distribution for commercial purposes requires written permission. labeling pattern in the mushroom bodies, the central complex, the dorsal lobe, and the gnathal ganglia, indicating a conserved receptor expression. With respect to sucrose responsiveness this result is of special importance. We assume that the tyramine 1 receptor expression in these neuropiles provides the basis for modulation of sucrose responsiveness. Furthermore, the tyramine 1 receptor expression seems to be independent of size, as labeling is similar in bee species that differ greatly in their body size. However, the situation in the optic lobes appears to be different. Here, the lobula of stingless bees is clearly labeled by the tyramine receptor 1 antibody, whereas this labeling is absent in other species. This indicates that the regulation of this receptor is different in the optic lobes, while its function in this neuropile remains unclear.

(C) 2021 The Author(s)

Published by S. Karger AG, Basel

\section{Introduction}

Eusociality is the highest level of social organization. It is characterized by: (1) cooperative brood care with only a small number of individuals in a colony being fertile, (2) 
common food procurement, and (3) the coexisting of multiple generations. Eusocial insects must coordinate the many different tasks in a colony as effectively as possible among sometimes tens of thousands of individuals. For this purpose, they have evolved intricate mechanisms of division of labor. An important hypothesis for understanding this division of labor is based on differences in behavioral response thresholds [Robinson, 1992]. Individuals that are involved in different tasks have different response thresholds for task-associated stimuli. The individual with the lowest thresholds for a given task is the first to perform it, while stimulus intensity needs to increase before other individuals with higher response thresholds for this stimulus will engage in this task. This theory has been studied in detail in the Western honeybee Apis mellifera. Here, it was demonstrated that individuals performing different tasks have different response thresholds for the stimulus sucrose. This sugar is the most important carbohydrate source for honeybees and a major component of nectar. Nurse bees, which provide larvae with protein-rich food, have generally a lower responsiveness to sucrose than forager bees, which collect pollen and nectar outside the hive [Thamm and Scheiner, 2014; Thamm et al., 2018]. Similarly, same age nurses and foragers in a single cohort exhibit respective differences in the sugar thresholds ("single cohort colony" [Huang and Robinson, 1992]).

The biogenic amine tyramine is generally considered as a modulator of sucrose responsiveness. The tyramine content in the brain correlates with the social role, that is, nurse bees have lower tyramine titers than forager bees [Scheiner et al., 2017a]. Increasing tyramine titers (by feeding or injection) lowers the response thresholds for sucrose [Scheiner et al., 2002, 2017a] and this effect can be blocked pharmacologically [Scheiner et al., 2017a] by the tyramine receptor inhibitor yohimbine [Reim et al., 2017]. Tyramine mediates its effect by binding to G-protein-coupled receptors (GPCRs), which initiates intracellular second messenger cascades (for a review see Finetti et al. [2021]). In the honeybee genome, 2 tyramine receptor genes encode specific tyramine GPCRs which upon activation lead to either an intracellular cAMP increase or decrease [Blenau et al., 2000; Reim et al., 2017]. Here, the gene Amtar1 (A. mellifera tyramine receptor 1 gene) is of particular interest. Two genetically different honeybee lines which differ in their responsiveness to sucrose [Scheiner et al., 2001] were analyzed using quantitative trait loci mapping (QTL mapping). The 2 lines differ significantly in several QTLs, with $p \ln 2$ being particularly noteworthy since it contains the Amtarl gene [Hunt et al., 2007]. Thus, pln 2 further corroborates the hypothesis that the respective tyramine receptor might be involved in the regulation of sucrose responsiveness. Finally, we developed a specific polyclonal antibody to investigate the expression of this tyramine receptor (AmTAR1) in the brain of A. mellifera [Thamm et al., 2017]. We found AmTAR1-like immunoreactivity (AmTAR1-IR) in the antennal nerve, the dorsal lobe, and the gnathal ganglion [Thamm et al., 2017]. These brain areas are crucial for the transmission and processing of gustatory and mechano-sensory information from the antenna [Haupt, 2007]. Furthermore, Jain and Brockmann [2020] found Amtar1 expression in the antennae. Thus, the expression of AmTAR1 in these structures is the basis for tyraminergic modulation of sucrose responsiveness. In addition, AmTAR1-IR was found in the mushroom bodies and the central complex [Thamm et al., 2017]. Both are brain regions which integrate multi-sensory information and play a role in decision making and foodreward dependent learning and memory [Barron et al., 2015].

Currently, it is unknown if and to what extent AmTAR1 expression in the brain of bees with different social organization, from solitary to eusocial living, is conserved. This is especially true for the brain areas that are relevant for the perception and evaluation of gustatory information. Thus, the question arises of whether the TAR1 expression is a special adaptation of the Western honeybees, A. mellifera, or if it represents a conserved trait among social and solitary bees for the purpose of modulating reaction thresholds for sucrose. To answer this question, we compared the spatial expression of the tyramine 1 receptor in the brain of different corbiculate bee species, with sucrose being an essential carbohydrate source for all of these species. Those include honeybees (Apini), bumblebees (Bombini), and stingless bees (Meliponini), using our AmTAR1-specific antibody. Representatives of this monophyletic group are solitary or show different degrees of eusociality. Based on a variety of different markers, the phylogenetic relationships within this group have been debated controversially (summarized in Kawakita et al. [2008]). However, the phylogenetic analyses of molecular markers suggest that the eusocial honeybees together with the solitary or communal orchid bees (Euglossini) form the sister group of the primitively eusocial bumblebees and the eusocial stingless bees [Kawakita et al., 2008, Rodriguez-Serrano et al., 2012]. Consequently, eusociality probably developed several times independently in this group [Rodriguez-Serrano et al., 2012]. In contrast, the 
Table 1. Information about the bee species analyzed in this study

\begin{tabular}{|c|c|c|c|c|}
\hline Family & Tribus & Species & Sampling site & Specimen No. \\
\hline \multirow[t]{9}{*}{ Apidae } & \multirow[t]{3}{*}{ Apini } & Apis dorsata & NCBS Campus, Bangalore, IND & 8 \\
\hline & & Apis cerana & NCBS Campus, Bangalore, IND & 11 \\
\hline & & Apis florea & NCBS Campus, Bangalore, IND & 9 \\
\hline & Bombini & Bombus terrestris & University of Würzburg, GER & 6 \\
\hline & \multirow[t]{5}{*}{ Meliponini } & Melipona costaricensis & Research station La Gamba, CR & 5 \\
\hline & & Tetragona ziegleri & Research station La Gamba, CR & 8 \\
\hline & & Partamona orizabaenzis & Research station La Gamba, CR & 5 \\
\hline & & Trigona fulviventris & Research station La Gamba, CR & 8 \\
\hline & & Trigonisca pipioli & Research station La Gamba, CR & 5 \\
\hline Megachilidae & Osmiini & Osmia bicornis & University of Würzburg, GER & 6 \\
\hline
\end{tabular}

CR, Costa Rica; GER, Germany; IND, India.

Euglossini formed the most basal group of the corbiculate bees in a more recent study [Bossert et al., 2019]. We additionally analyzed the TAR1 distribution in solitary Osmia bicornis which belongs to the Megachilidae. This family is formed by mostly solitary and nest-building bees [Gonzalez et al., 2012] and is closely related to the Apidae [Branstetter et al., 2017; Sann et al., 2018]. Additionally, several different species will be investigated in the group of highly eusocial stingless bees. Their body size varies from approximately $11 \mathrm{~mm}$ body length for Trigona and Melipona species (corresponding approx. to A. mellifera) down to approximately $2.5 \mathrm{~mm}$ body length for, for example, Trigonisca species. Thus, we can additionally analyze whether brain miniaturization processes influence the spatial TAR1 expression in specific brain areas. Finally, this analysis enables us to compare the spatial TAR1 brain expression of a well-established model organism with that of closely related non-model species and will be an important step forward to understanding the functions of tyramine in behavioral processes of insects.

\section{Materials and Methods}

\section{Animals}

The species that were analyzed in this study are summarized in Table 1. Worker bees of the different honeybee species (A. cerana, A. dorsata, A. florea) were collected from colonies hosted on NCBS campus. Workers of Bombus terrestris were taken directly from commercial colonies at the University of Würzburg (kindly provided by Johannes Spaethe). Stingless bees were captured at their hive entrance or while foraging at flowers or at artificial feeders. $O$. bicornis were purchased commercially.

TAR1 Brain Distribution in Corbiculate Bees

\section{Western Blot}

Brains of B. terrestris and O. bicornis were dissected in ice-cold phosphate-buffered saline (PBS; $140 \mathrm{mM} \mathrm{NaCl}, 2.7 \mathrm{mM} \mathrm{KCl}, 10$ $\mathrm{mM} \mathrm{Na}_{2} \mathrm{HPO}_{4}, 1.8 \mathrm{mM} \mathrm{KH}_{2} \mathrm{PO}_{4}, \mathrm{pH} 7.3$ ). Directly after the preparation, cytosolic and membrane proteins were extracted and subsequently analyzed by immunoblotting, as described in Thamm et al. [2017].

\section{Immunohistochemistry}

Captured bees were immediately anesthetized on ice, and their brains were dissected and fixed in Bouin-Hollande fixative $(0.04 \mathrm{M}$ picric acid, $0.125 \mathrm{M}$ copper(II) chloride, $0.125 \mathrm{M}$ sodium acetate, $11 \%(\mathrm{v} / \mathrm{v})$ acetic acid, $0.33 \%(\mathrm{v} / \mathrm{v})$ formaldehyde) over night. Thereafter, the brains were washed with $70 \%(\mathrm{v} / \mathrm{v})$ ethanol 3 times. Finally, brains were stored in $70 \%(\mathrm{v} / \mathrm{v})$ ethanol until further processing. After rehydration (60, 40, 20, 0\% [v/v] ethanol in PBS), the brains were embedded in 5-7\% agarose and sliced using a vibratome. Afterwards, these slides were labeled with a specific antibody against the honeybee tyramine receptor 1 (anti-AmTAR1, Thamm et al. [2017]). This antibody is directed against the cytoplasmic loop 3 of the receptor protein, whose amino acid sequence is highly conserved among closely related species (Fig. 1). The labeling procedure was carried out as described for A. mellifera [Thamm et al., 2017]. Merely the thickness of the slides was adjusted to the size of the brain (Table 1).

\section{Image Acquisition}

All selected brain slices were imaged by laser-scanning confocal microscopy using a Leica TCS SP2 AOBS (Leica Microsystems AG, Wetzlar, Germany) equipped with an argon/krypton laser and 3 diode lasers. HC PL APO objective lenses $(10 \times / 0.4$ NA imm; $20 \times / 0.7 \mathrm{NA}$ imm, and $63 \times / 1.20 \mathrm{NA} \mathrm{imm}$ ) with additional digital zoom were used for image acquisition. Finally, the images were processed with Image J (version 1.52p) and Inkscape (version 1.0.1) was used to arrange the images into figures. 


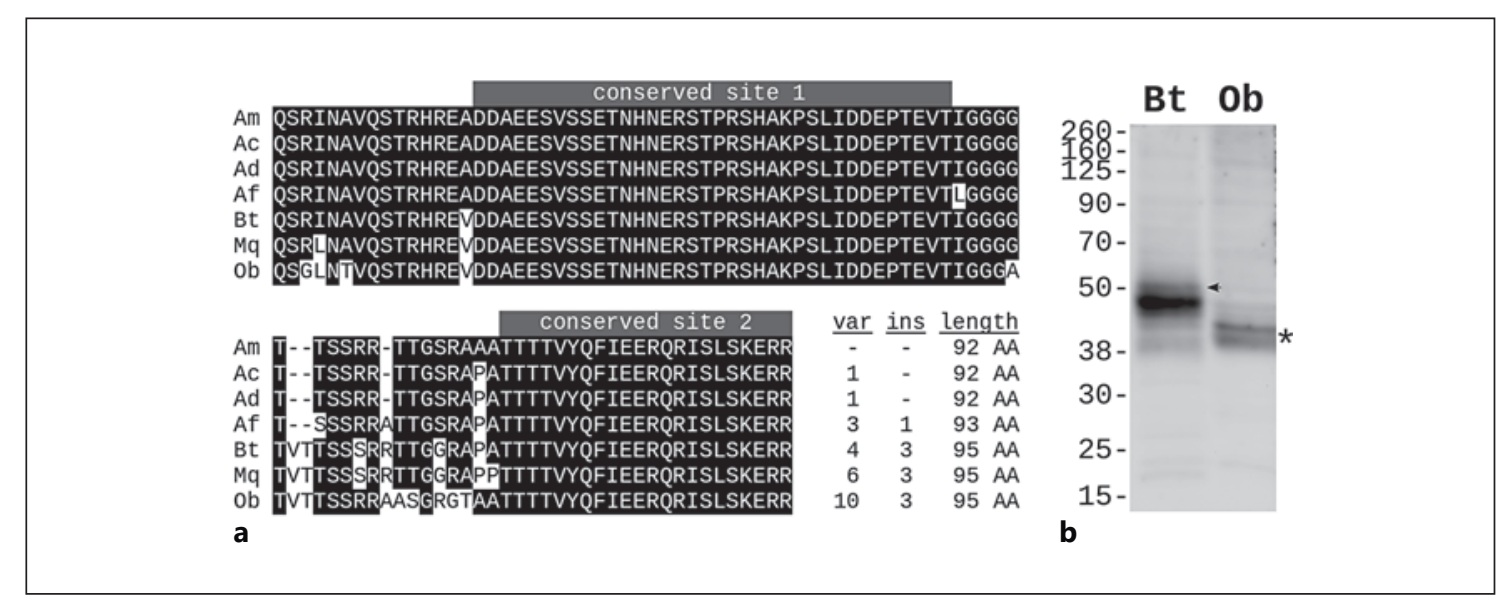

Fig. 1. Sequence comparison of the TAR1 antigen and Western blot analysis with the anti-AmTAR1 antibody. a Alignment of the amino acid sequence of that part of the cytoplasmic loop 3 of AmTAR1, which served as the antigenic determinant (see Thamm et al. [2017]), and the deduced amino acid sequence of the same region of the orthologous receptor from other hymenopteran species analyzed in this study or closely related species. Only a small number of variable sites (var) and insertions (ins) occur among this specific part of the receptor protein, even in the out group species $O$.

\section{Results}

\section{Comparison of the Antigenic Determinant}

As described in Thamm et al. [2017], a specific part of the third cytoplasmic loop of AmTAR1 was used for the generation of a specific antibody. To estimate the likelihood that this antibody will recognize TAR1 not only in A. mellifera, we compared the deduced amino acid sequence of the antigenic determinant for the species that were analyzed in this study or, if the sequence information was not available, in closely related species. In general, the third cytoplasmic loop (CPL3) is conserved well, in which 2 sites are 100\% identical (conserved site 1 and 2 in Fig. 1a). Besides this, only a few variable sites and insertions can be detected. Here, the CPL3 of TAR1 of $M$. quadrifasciata, which represents a stingless bee, showed less conservation (6 variable sites and 3 insertions, Fig. 1a) among the corbiculate bees. Similarly, the CPL3 of TAR1 in O. bicornis did not show much more variation (10 variable sites and 3 insertions, Fig. 1a).

\section{Western Blot Experiments}

Western blot experiments with brains of $B$. terrestris and $O$. bicornis were carried out to analyze whether the AmTAR1 antibody is able to recognize protein bands of the expected molecular weight in a broader range of bee bicornis. Am, A. mellifera (XP_026295054.1); Ac, A. cerana (XP_016921562.1); Ad, A. dorsata (WJNQ01005242.1); Af, A. florea (XP_012348058.1); Bt, B. terrestris (XP_012175128.2); Mq, M. quadrifasciata(LIRP01011901.1);Ob,O.bicornis(XP_029051699.1). b Western blot of membrane proteins of brains of $B$. terrestris $(\mathrm{Bt})$ and $O$. bicornis $(\mathrm{Ob})$. In $B$. terrestris a strong protein band of approximately $45 \mathrm{kDa}$ and a slightly larger but much weaker band (arrow) appeared. In O. bicornis we could detect 2 protein bands very close to each other (asterisk) of approximately $40 \mathrm{kDa}$.

species. In A. mellifera brain homogenates the antibody recognizes a protein of approximately $45 \mathrm{kDa}$ [Thamm et al., 2017]. In bumblebee brain homogenates, a strong protein band of approximately $45 \mathrm{kDa}$ appears (Fig. 1b). Additionally, a slightly bigger protein is labeled (arrow in Fig. 1b). Both proteins correspond to the predicted molecular weight of both annotated isoforms of TAR1 in this species, X1 (55.6 kDa, XP_012175128.2) and X2 (44.9 $\mathrm{kDa}, \mathrm{XP}$ _012175179.1). In O. bicornis brains we could also detect 2 labeled protein bands (Fig. 1b). However, they are smaller than the expected molecular weight of the receptor (44.8 kDa, XP_029051699.1).

\section{Immunohistochemistry Overview}

In general, the labeling of brains of the different bee species (for the number of specimens/species see Table 1) with the anti-AmTAR1 antibody produced very similar results. For an overview of the TAR1-like immunoreactivity (TAR1-IR) for each species, see the online supplementary Figures S1-S10 (for all online suppl. material, see www.karger.com/doi/10.1159/000517014). Intriguingly, the labeling of the anti-AmTAR1 antibody seems weaker in O. bicornis brains and thus an overall higher background signal was observable. In all species, we found similar TAR1-IR in the mushroom bodies, the dorsal lobe and in the gnathal ganglia. However, we also
16

Brain Behav Evol 2021;96:13-25 DOI: $10.1159 / 000517014$ 

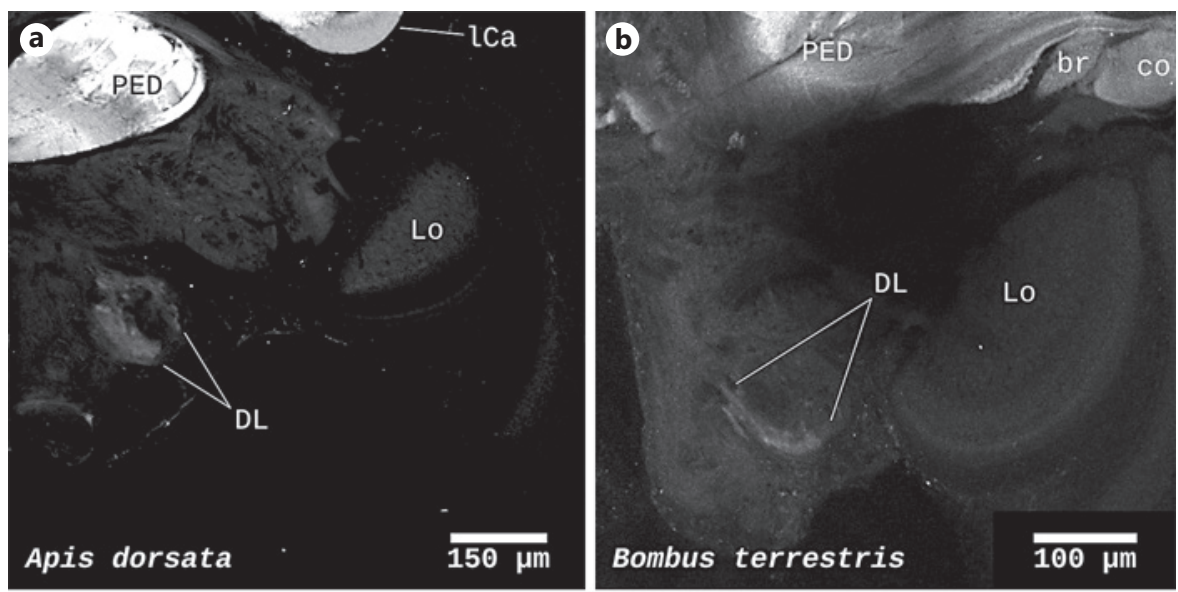

Fig. 2. TAR1-IR in the dorsal lobe. Frontal brain sections show TAR1-IR labeling in the dorsal lobe of $A$. dorsata (a), B. terrestris (b), T. pipioli (c), and O. bicornis (d). br, basal ring; co, collar; DL, dorsal lobe; $\mathrm{lCa}$, lateral calyx; Lo, lobula; Me, medulla; PED, peduncle.
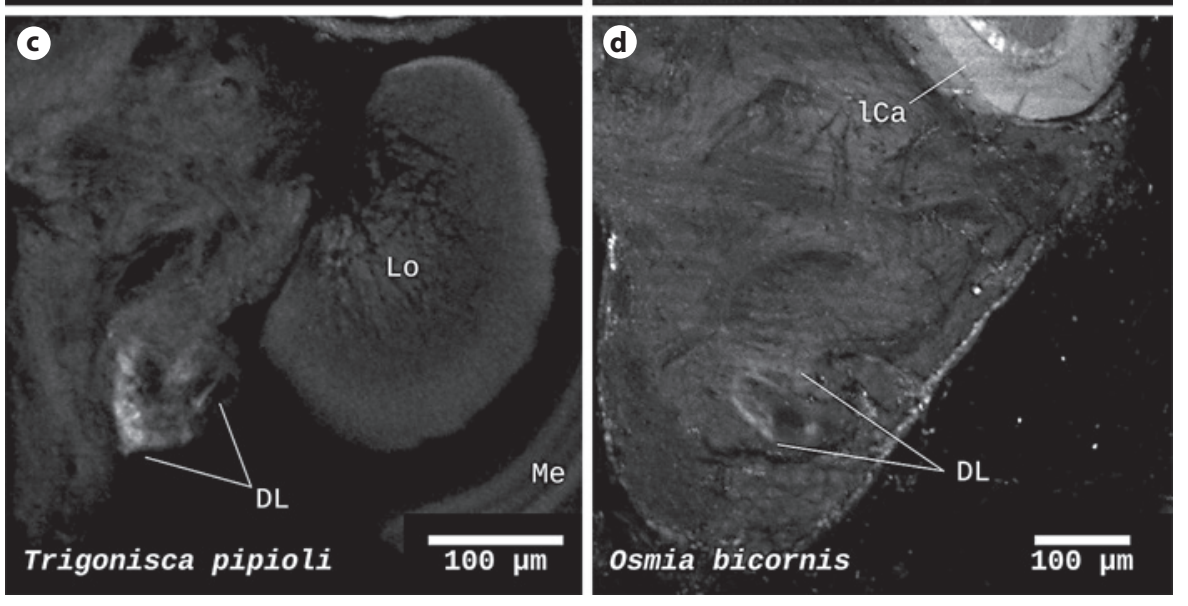

found 2 major exceptions. First, clear TAR1-IR can also be found in the optic lobes of stingless bees and of $\mathrm{O}$. $\mathrm{bi}$ cornis. Second, TAR1-IR is observable in some neurons (mainly the cell body) in O. bicornis brain. The following sections will describe these results in more detail. For a more detailed description of the different brain parts in A. mellifera, see Thamm et al. [2017] or the more specific literature [Kenyon, 1896; Ehmer and Gronenberg, 2002; Strausfeld, 2002; Kirschner et al., 2006].

\section{Antennal Lobe, Dorsal Lobe, and Gnathal Ganglia}

In all bee species in this study, we can observe TAR1IR in the dorsal lobe (details: Fig. 2a-d; overview: online suppl. Fig. S1-10) which is the neuropile that is important in the processing of gustatory and mechano-sensory information from the antennae [Haupt, 2007]. In some preparations, further TAR1-IR can be observed in distinct axonal bundles within the antennal nerve that project into the dorsal lobe (online suppl. Fig. S7, S9). In contrast, we observe no TAR1-IR in the antennal lobe, where olfactory signals are processed [Sandoz, 2012]. However, in some rare preparations a weak labeling of the glomeruli is observable (online suppl. Fig. S7, S10).

Additionally, TAR1-IR-positive paired structures are observable in the gnathal ganglion of Apini and Meliponini, as exemplarily shown for A. dorsata (Fig. 3a) and T. pipioli (Fig. 3c). Those structures are likely connected to sensilla on the head capsule via the tegumentary nerve, which is also TAR1-IR positive (online suppl. Fig. S1, S6). In $B$. terrestris and O. bicornis, similar structures are hard to find because of weak labeling (Fig. 3b, d).

\section{Optic Lobes}

In the optic neuropils of the Apini and the Bombini we could not detect any TAR1-IR, or only very weak labeling (Fig. 4a, b). This is also true for the Meliponini in the case of medulla and lamina. However, in the lobula of the investigated stingless bee species we have another picture. Here, we observed TAR1-IR in most lateral layers of this neuropile as exemplarily shown for T. fulviventris (arrows in Fig. 4c) and T. pipioli (arrows in Fig. 4d). In some preparations (but not in all) we can additionally observed a 

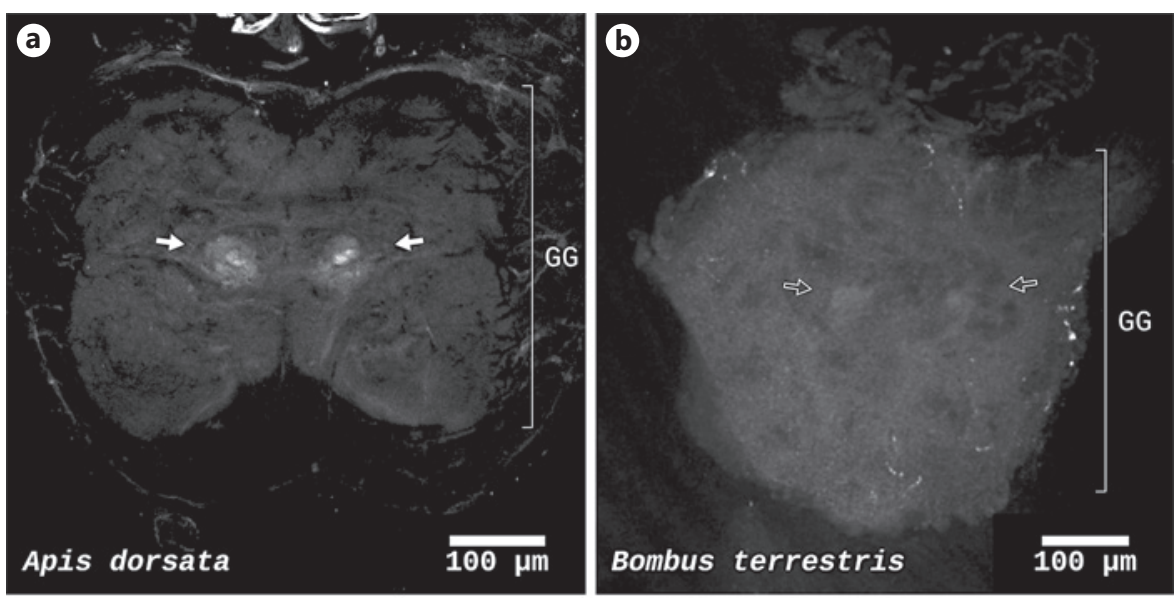

Fig. 3. TAR1-IR in the gnathal ganglion (GG). Frontal brain sections show paired structures (filled arrows) with TAR1-IR labeling in gnathal ganglia of $A$. dorsata (a) and T. pipioli (c). In B. terrestris (b) and $O$. bicornis (d) similar labeling is hard to find because it is much weaker (unfilled arrows).
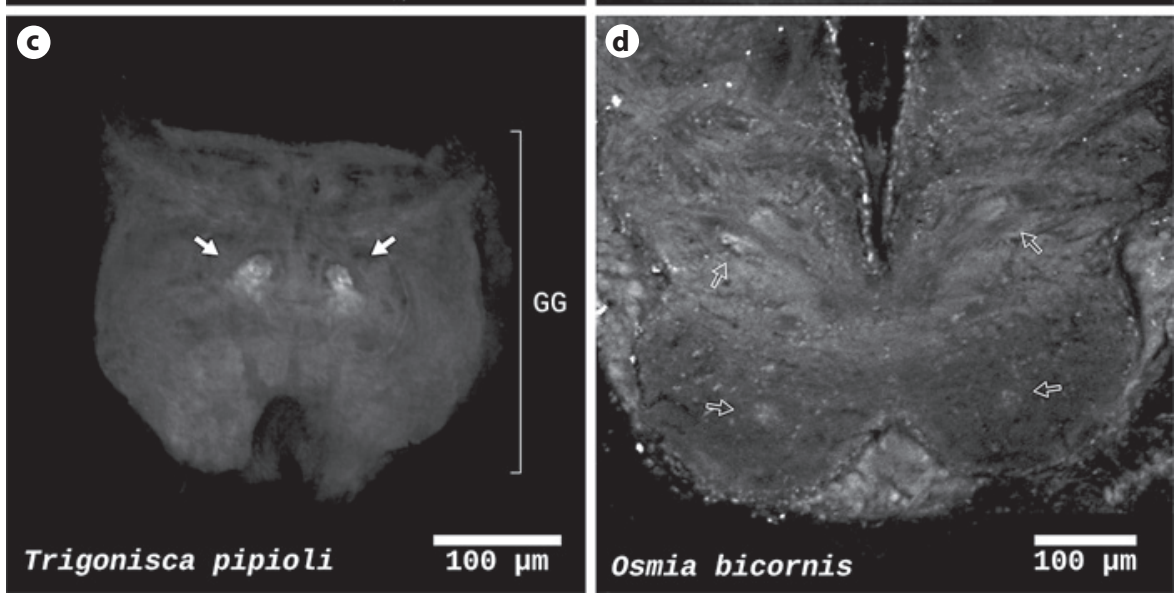

TAR1-like positive neuronal tract from the lobula into a ventro-lateral neuropile in the brain. This is particularly noticeable in preparations of T. fulviventris (asterisk in Fig. 4c). Similar labeling of the lobula is observable in $O$. bicornis (arrow in Fig. 4d).

\section{Mushroom Bodies}

A very prominent TAR1-like immunohistochemistry is observable in the mushroom bodies of all examined bees in this study (Fig. 5). The lip, collar, and basal ring exhibit intense TAR1-like labeling. The most intensive signal was shown by the basal ring (Fig. 5a-c). Only in $O$. bicornis is this labeling comparatively weak (Fig. 5d). Whereas olfactory projection neurons (PNs) branch in the lip, visual PNs end in the collar, multisensory PNs in the basal ring, and gustatory $\mathrm{PNs}$ branch in a small region between lip and collar [Rybak and Menzel, 2010].

Strong TAR1-like labeling can also be found in several fibers in the peduncles in all species (Fig. 5a-d). The Kenyon cells send their axons to the output regions of the mushroom bodies via the peduncles. The output regions consist of the medial lobe (Fig. 5a-d), the vertical lobe, and the $\gamma$-lobe (Fig. 5e-h). All of these show TAR1-IR with variable intensities. Here, 2 regions with stronger TAR1-IR are protruding. One can be found in the vertical lobe (asterisks in Fig. 5e-g) and the other in the $\gamma$-lobe (\# in Fig. 5e-g). The cell somata of the Kenyon cells, however, only show weak labeling, although labeling is not absent here. Some TAR1-IR-positive fibers can be found in the boundary layer between the densely packed type I Kenyon cell somata in the center (supplied by the collar and lip region) and the larger and more loosely packed type I Kenyon cell somata in the periphery of the calyx (arrow in Fig. 5a, b). In the smaller species of stingless bees, those fibers seem to be located not only in that boundary layer, but also in the center of the calyx (asterisk in Fig. 5c). In O. bicornis, a labeling of those fibers is weak but still observable (Figure Fig. 5d, 6a).

\section{Central Complex}

In the central complex of Apini, Bombini, and Meliponini we can observe TAR1-IR in the so-called ellipsoid 


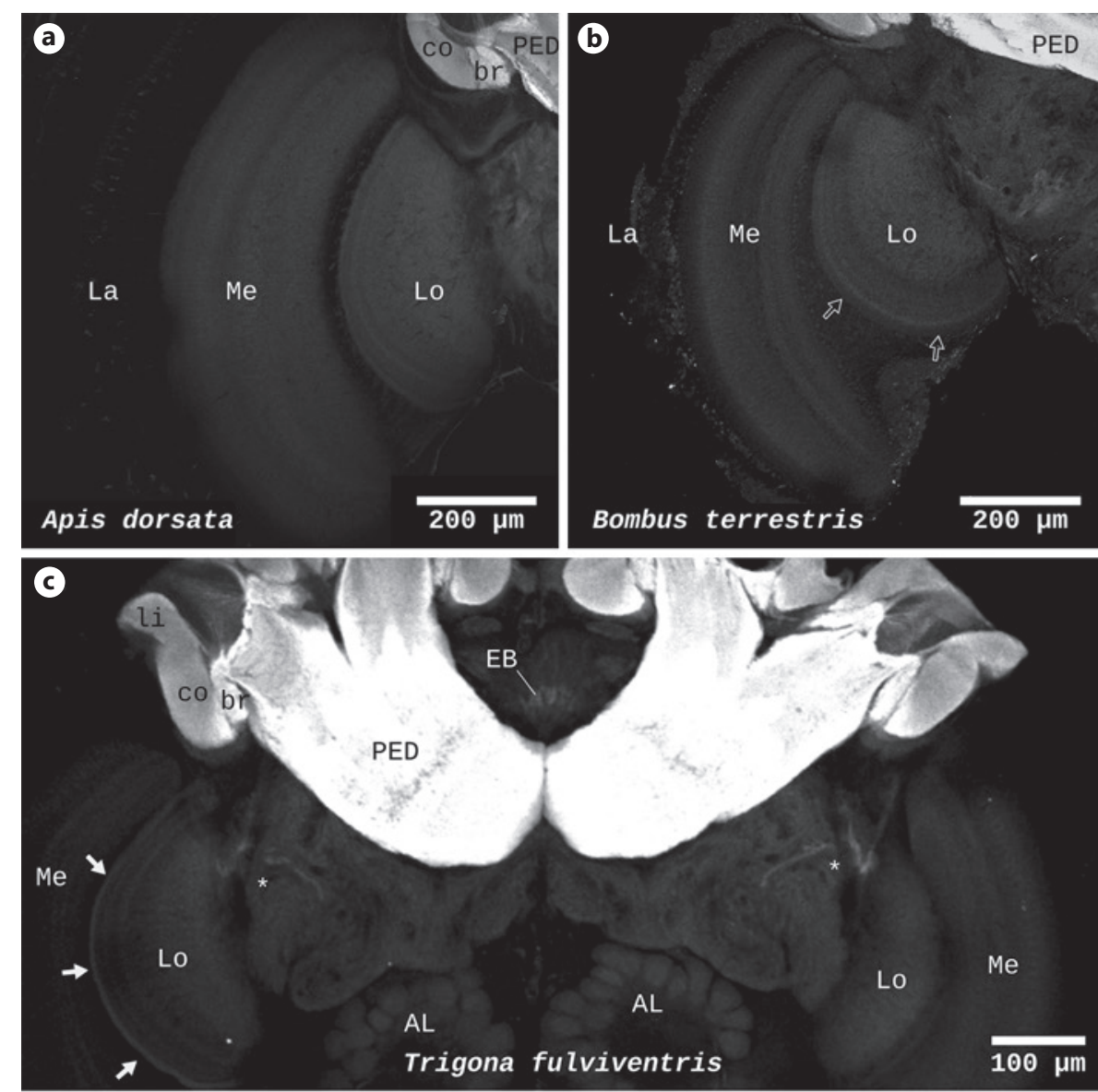

Fig. 4. TAR1-IR in the optic lobes. Frontal brain sections of different bee species were treated with the AmTAR1 antibody. In the optic lobes of $A$. dorsata (a) no obvious TAR1-IR was detectable. In B. terrestris (b) we found a weak labeling of the most lateral layer of the lobula (unfilled arrows). A similar but much clearer labeling (filled arrows) appears in T. fulviventris (c), T. pipioli (d), and O. bicornis (e); additionally, TAR1-IR-positive nerve-like structures (asterisks) run from the lobula into a ventro-lateral neuropile in the brain of T. fulviventris (c). AL, antennal lobe; br, basal ring; co, collar; DL, dorsal lobe; li, lip; lCa, lateral calyx; La, lamina; Lo, lobula; Me, medulla; PED, peduncle.
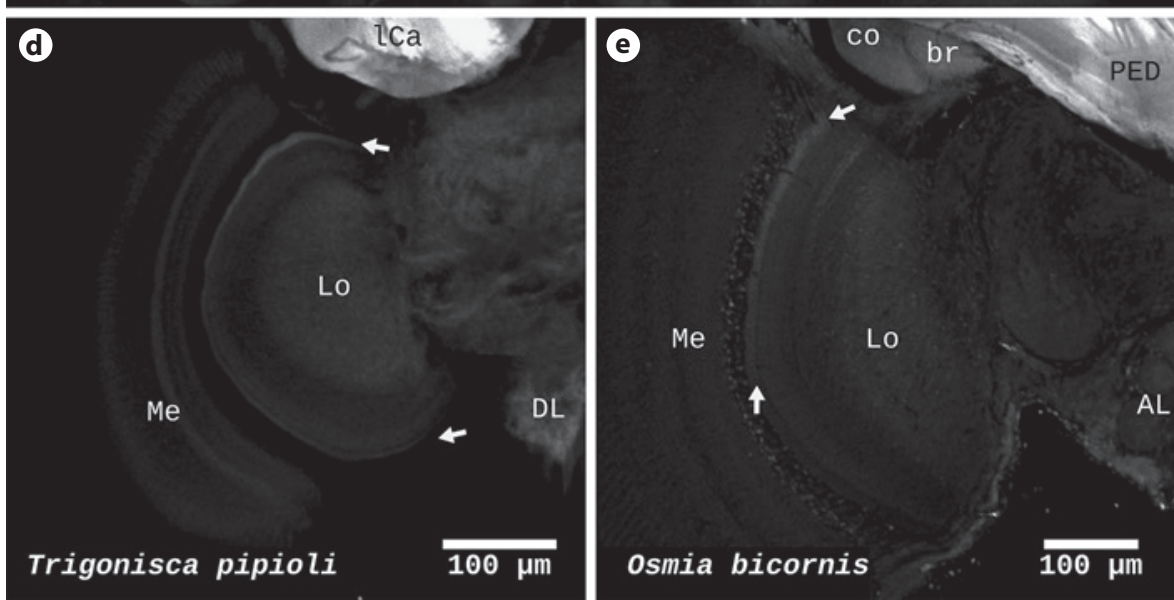

body (EB) of the central body (Fig. 5a-c), whereas the fan-shaped body (FB) and the proto-cerebral bridge remained unlabeled. In addition, the medial and lateral bulbs (lBU and $\mathrm{mBU}$ ) are TAR1-IR positive (e.g., $\mathrm{mBU}$ in Fig. 5b). We also observed a labeling of those structures in O. bicornis. Here, the FB is labeled additionally and the TAR1-IR intensity of EB and FB is nearly the same (Fig. 5a; online suppl. S10).

TAR1 Brain Distribution in Corbiculate Bees
Cell Bodies in the Brain of O. bicornis

We further found clear TAR1-IR in some cell bodies in O. bicornis which are absent in the brains of the other bees (Fig. 6). This labeling differs from the results we described above where the cell body is clearly not labeled. In addition, the position of the cell nucleus (which is not stained) is clearly visible, which indicates a cytosolic labeling.

Brain Behav Evol 2021;96:13-25 

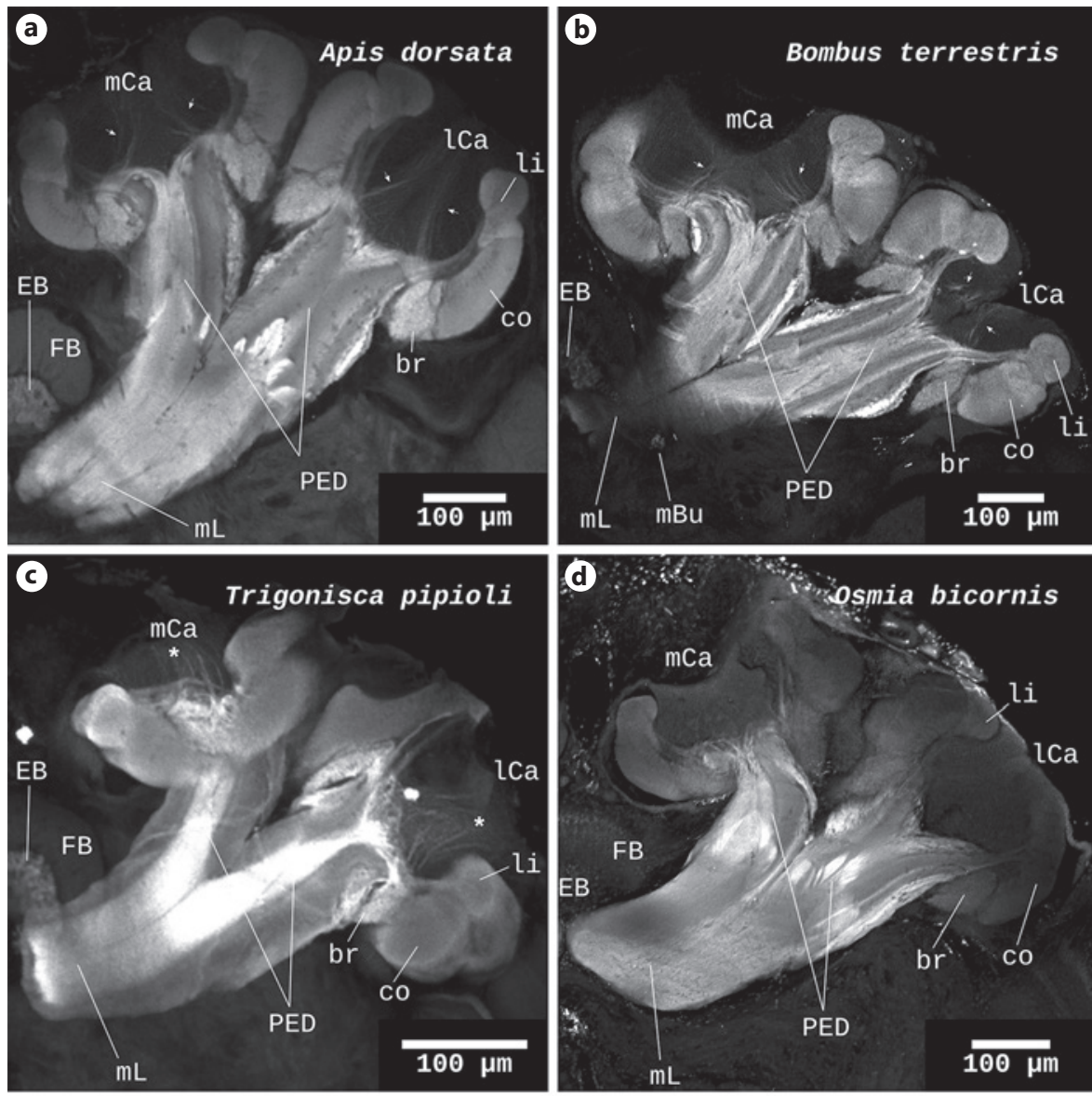

Fig. 5. TAR1-IR in the mushroom bodies and central complex. Frontal brain sections of $A$. dorsata (a, e), B. terrestris (b, f), $T$. pipioli (c, g), and O. bicornis $(\mathbf{d}, \mathbf{h})$ were treated with the anti-AmTAR1 antibody. a-d TAR1-IR in the calyces, peduncles, and medial lobes of the mushroom bodies and in the central complex. e-h Strong TAR1-IR was also found in the vertical and the $\gamma$-lobe of the mushroom bodies. br, basal ring; mCA, medial, calyx; co, collar; EB, ellipsoid body; FB, fan-shaped body; mL, medial lobe; lCa, lateral calyx; li, lip; PED, peduncle; vL, vertical lobe; $\gamma \mathrm{L}, \gamma$-lobe.
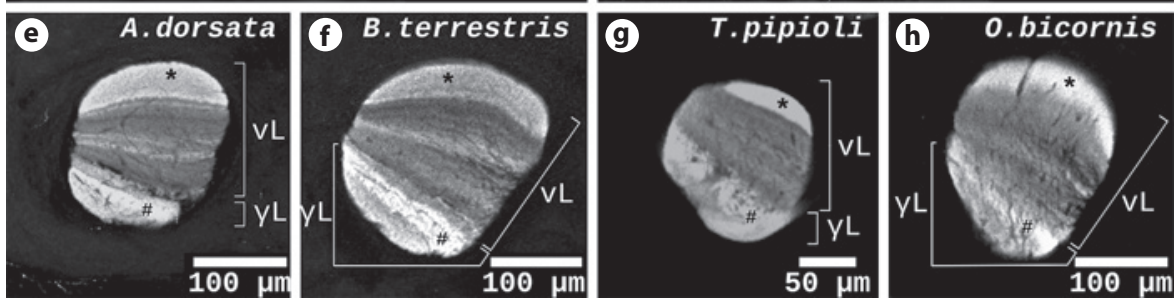

\section{Discussion}

With this study, we compared the spatial expression of the tyramine 1 receptor in the brain of the well-studied Western honeybee A. mellifera with that of closely related non-model species. For these analyses we used a polyclonal antibody against a specific part of the honeybee orthologue of this receptor (AmTAR1, see Thamm et al. [2017]). The overall labeling pattern in the brain of honeybees, bumblebees, and stingless bees is very similar, if not identical. This comprises labeling of the mushroom bodies, the central complex, the dorsal lobe, and the gnathal ganglia. The only exception is the labeling of the lobula (and partially the medulla) and corresponding ventro- lateral neuronal tracts in brains of stingless bees, which we do not find in honeybee and bumblebee brains and which are discussed in detail below. We are convinced that our TAR1 receptor antibody is suited for a comparative analysis among corbiculate bees for different reasons. The corresponding antigen is highly conserved within the corbiculate bees. Within this antigen we found 2 sites of 36 and 22 amino acid lengths that are completely conserved and beyond that only few variable sites and insertions can be detected (Fig. 1a). Although there are 4 variable sites and 3 insertions in the antigenic region in TAR 1 of $B$. terrestris when compared with the same region in A. mellifera, we can detect protein bands in the membrane protein fraction of bumblebee brain lysates in Western blot experi- 
Fig. 6. a, b TAR1-IR in neuronal cell bodies in O. bicornis. Frontal brain section treated with the anti-AmTAR1 antibody. At least one cluster of 3-5 cells with TAR1-IR can be found between the 2 medial calyces of the mushroom bodies. The localization of the nucleus in those cells is obvious (no labeling). Thus, it probably represents a cytosolic labeling. br, basal ring; co, collar; li, lip; VL, ventral lobe.
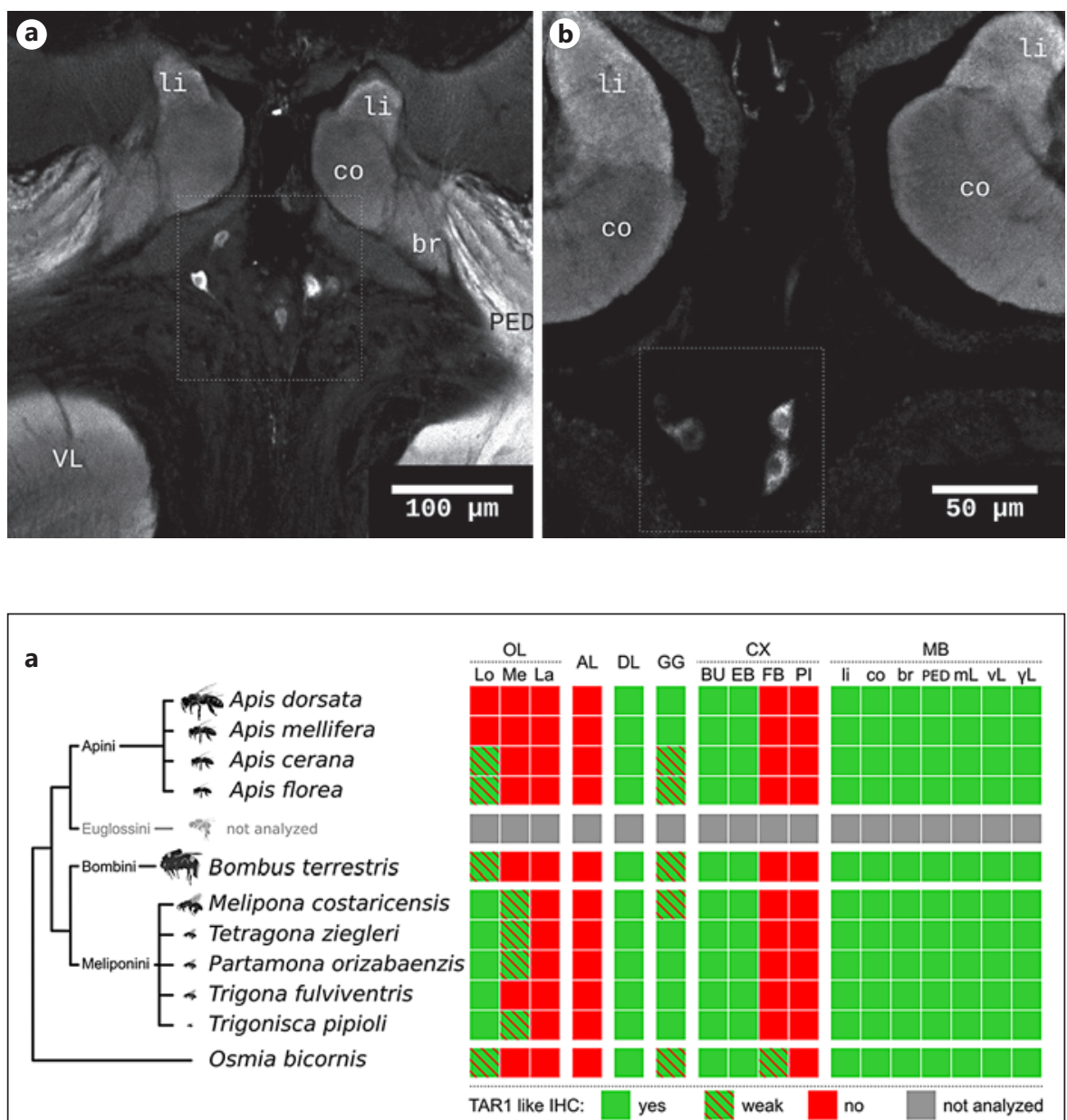

Fig. 7. TAR1-IR overview in the brain of corbiculate bees. a Schematic overview of the TAR1-IR in the brain of the corbiculate bees. b Brain size comparison of a large (left) and a small stingless bee (right). AL, antennal lobe; BU, bulbs; br, basal ring; CX, central complex; co, collar; DL, dorsal lobe; EB, ellipsoid body; FB, fan-shaped body; GG, gnathal ganglion; La, lamina; Lo, lobula; li, lip; MB, mushroom bodies; Me, medulla; mL, medial lobe; OL, optic lobes; PED, pedunculus; PI, pars intercerebralis; $\mathrm{vL}$, ventral lobe; $\gamma \mathrm{L}, \gamma$-lobe.

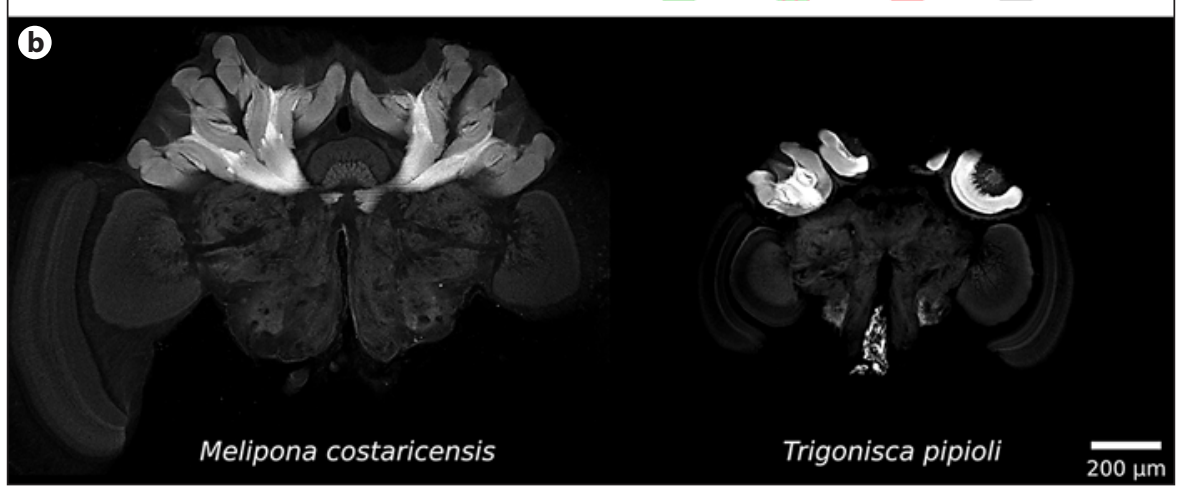

ments that correspond to the predicted molecular weight of the receptor (Fig. 1b). These results are comparable to Western blot experiments conducted in A. mellifera [Thamm et al., 2017]. We therefore assume that the polyclonal AmTAR1 antibody recognizes TAR1 across species. However, this assumption appears less appropriate when the species are phylogenetically more distant, such as the solitary megachilid bee $O$. bicornis. Although the 2 conserved sites in the antigenic determinant are con- served as well, we found considerably more variation (see Fig. 1a). Furthermore, in Western blots we detected a protein with a lower molecular weight (approx. $40 \mathrm{kDa}$ ) than that of the annotated O. bicornis TAR1 (44.8 kDa). Also, in this species we do not have the same quality of TAR1IR as in corbiculate bee species. Although the same structures are labeled as mentioned above (mushroom bodies, central complex, dorsal lobe, gnathal ganglia, lobula), the labeling seems much weaker and background signals are 
much stronger. One detail in the brain of O. bicornis was particularly surprising. Beside the TAR-IR described above, we found additional cell bodies with extensive TAR1-IR. Comparable results could not be obtained in the brain of A. mellifera [Sinakevitch et al., 2017; Thamm et al., 2017] or in the brains of the other bees in the present study. In terms of quality, these cell body labels also differ from the rest of the TAR1-IR: in contrast to the results described above, our antibody appears to recognize proteins in the cytoplasm rather than in the membrane. It is conceivable that a membrane protein is detectable at endoplasmic reticulum if translation takes place at high throughput or if it is stored in the lysozyme. So far, the reason why these cells behave completely differently to the majority of cells with positive TAR-IR has remained unclear to us. The most plausible explanation for this labeling is that it does not represent TAR1 expression. In summary, we must state that the results of $O$. bicornis have to be interpreted with utmost caution, given that some of the described immunolabeling may be unspecific.

Tyramine is assumed to be an important modulator of the processing and/or integration of sensory information in insects [Nisimura et al., 2005; Scheiner et al., 2017a; Damrau et al., 2018]. Since such a function is reported from diverse insects from a wide range of insect orders, this points to a conserved feature of this neuromodulator. Such a conservation in function can be achieved, for example, via the conservation of the receptor expression in the brain structures responsible for the respective sensory processing and integration. Interestingly, our analysis shows that TAR1-IR is highly conserved in the brain structures that are important for gustatory information processing and sensory integration. These findings suggest that this tyramine receptor has similar functions across bee species.

First, TAR1-IR was found in the antennal nerve, the dorsal lobe, the gnathal ganglia, and in some preparations also in the tegumentary nerve, as was shown before for $A$. mellifera [Thamm et al., 2017]. Here, the antennal nerve (or parts of it) and the dorsal lobe are of special interest. These structures are important as signals that are perceived at the antennae and processed in the brain have to pass through these neuropils [Brockmann and Robinson, 2007; Haupt, 2007; Jain and Brockmann, 2020]. During that process it is likely that the signals are modulated by the activation of TAR1. Indeed, in A. mellifera it was shown that tyramine can alter the responsiveness to sucrose [Scheiner et al., 2002] and that yohimbine, which is a potent TAR1 blocker [Reim et al., 2017], can block that action of tyramine [Scheiner et al., 2017a].
However, that effect of tyramine can also be the result of the modulation of gustatory signal integration in the mushroom bodies, as results from blowflies suggest. Here, tyramine modulates feeding thresholds that depend on the presence of non-appetitive and appetitive flavors, whereas tyramine has no effect on those thresholds in animals with ablated mushroom bodies [Nisimura et al., 2005]. This requires at least one tyramine-specific receptor subtype in the mushroom bodies, and previous studies in the Western honeybee A. mellifera suggested exactly this [Sinakevitch et al., 2017; Thamm et al., 2017]. In the other bee species, we found highly comparable and intense TAR1-IR in the mushroom bodies. The pattern of labeling seems to be highly conserved. The basal ring, for example, always shows strong TAR1-IR signals (Fig. 5ad) and the TAR1-IR pattern in the vertical and the $\gamma$-lobes are very similar (Fig. 5e-h). Finally, the idea of highly conserved TAR1 expression is supported by studies in Drosophila melanogaster and Periplaneta americana, whose mushroom bodies also express this receptor subtype [Rotte et al., 2009; El-Kholy et al., 2015].

Another important brain structure for sensory integration is the central complex, the main function of which is the integration of visual information and higher-order motor control [Strauss, 2002; Homberg, 2004; Pfeiffer and Homberg, 2014]. Concerning a conserved TAR1 expression, we have a similar situation as that in the mushroom bodies. Earlier studies showed that TAR1 is located in homologous structures: the EB of bees and flies and in the lower division of the central body in cockroaches [Rotte et al., 2009; El-Kholy et al., 2015; Sinakevitch et al., 2017; Thamm et al., 2017]. In the corbiculate bees, we saw the same picture: the EB was always TAR1-IR positive. Furthermore, the $\mathrm{mBU}$ and the $\mathrm{lBU}$ of the lateral complex are TAR1-IR positive, which was shown earlier in A. mellifera. Interestingly, in O. bicornis the FB of the central complex is labeled additionally. However, whether these signals indeed represent specific TAR1 labeling in this bee and whether TAR1 also has a conserved function in the modulation of visual information such as the sky compass [Pfeiffer and Homberg, 2014; Held et al., 2016] or is involved in motor control as suggested by results from bees and flies [Kutsukake et al., 2000; Saraswati et al., 2004; Fussnecker et al., 2006] has to be the subject of future studies.

The TAR1 expression in the brains of corbiculate bees seems to be highly conserved. Contrary to this finding, however, are the results from the optic lobes (Fig. 5). On the one hand, we cannot detect clear TAR1-IR in the optic neuropiles of the Apis species [this study; Thamm et 
al. 2017]. In the Meliponini, however, we found clear TAR1-IR in the lobula and in some species also some weaker labeling in the medulla. Although much weaker than in the stingless bees, similar labeling can be found in their sister group Bombini (B. terrestris). This points to a possible function of TAR1 in the modulation of motionsensitive signals that are processed in that neuropile. The Megachilidae and the Apidae likely represent sister groups. As already mentioned above, the antibody labeling in the brain of $O$. bicornis (Megachilidae) has to be interpreted with care. However, here a lobular TAR1-IR is also present. Assuming that this labeling is specific, TAR1 expression in the lobula possibly represents a basal feature within bees. Although no TAR1-IR was observable in the optic lobes of both A. mellifera nurse and forager bees, respectively, TAR1 expression in Apis species may be regulated more specifically dependent on factors that we do not understand currently. Indeed, earlier studies report mRNA expression of the respective receptor gene in the optic lobes of the Western honeybee [Blenau et al., 2000; Mustard et al., 2005; Reim et al., 2017]. We exclude masking due to fixation artifacts, because then we would expect a clear labeled protein band to appear in WB analyses of the optic lobes, which is not the case [Thamm et al. 2017]. However, we cannot rule out the possibility of endogenous processes that may explain the discrepancy of the results at mRNA and protein level. These include the modulation of transcription or translation, post-translational processing (that would mask the antigenic determinant), protein transport, and protein degradation [Vogel and Marcotte, 2012; Thamm et al., 2017; Patwardhan et al., 2021]. Further studies are necessary to solve this question.

One of our main questions was whether the brain TAR1 expression is a special adaptation of A. mellifera or whether it represents a conserved trait and possesses a special importance for the emergence of eusociality in corbiculate bees. The high degree of conservation of TAR 1 expression in the dorsal lobe and in the mushroom bodies indicates that the latter is the case. According to the response threshold theory [Robinson, 1992], there is a need for the modulation of sensory thresholds, which, in turn, determine the task a bee performs. As the nutrition of corbiculate bees relies on sugars, responsiveness to sucrose, which correlates with responsiveness to other sugars [Değirmenci et al., 2018], could be a key regulator of behavior. Tyramine was shown to affect sucrose responsiveness in A. mellifera [Scheiner et al., 2017a, b] and TAR1 is expressed in a highly conserved way in neuropiles which have an impact on sucrose responsiveness.

TAR1 Brain Distribution in Corbiculate Bees
Thus, we assume that TAR1 expression in the respective neuropiles provides the basis for sucrose responsiveness modulation. However, this cannot explain behavioral differences between individuals, as this requires differences between individuals that perform different tasks or species that differ in their social organization. One possible explanation could be that behavioral differences are regulated on the level of tyramine and/or tyramine release. Indeed, A. mellifera foragers have higher brain tyramine titers and a higher sucrose responsiveness than nurse bees [Scheiner et al., 2017a]. Additionally, nurse bees with artificially increased brain tyramine titers also display an increased sucrose responsiveness [Scheiner et al., 2017a]. To our knowledge, no studies are currently available that analyze tyramine effects on behavior in stingless bees and bumblebees. Only for octopamine are some data available. In M. scutellaris octopamine increases gustatory responsiveness similar to honeybees [McCabe et al., 2017]. In another stingless bee, Plebeia droryana, octopamine treatment increases the number of bees at artificial sucrose feeders, which was interpreted by an octopaminedriven lowering of the individual sucrose response threshold [Peng et al., 2020], similar to the effect observed in honeybees [Barron et al., 2002; Barron and Robinson, 2005]. However, these data suggest that modulation of sucrose responsiveness is likely more complex and, in addition to tyramine and its specific receptors, further modulators such as octopamine have to be considered. Our current data only allow us to speculate about the function of the tyramine receptor in the different bee species. Future experiments can help to understand the role of TAR1 but also the whole octopaminergic/tyraminergic system in modulating sucrose responsiveness and its impact on task allocation. Several approaches are conceivable, such as behavioral pharmacology [Scheiner et al., 2017a], gene knockdown using RNAi [Guo et al., 2018], or gene knockout with the CRISPR/Cas9 method [Değirmenci et al., 2020].

Another important aspect is that we have analyzed bees within a wide range of body sizes. These comprise huge bees like A. dorsata (17-20 mm), middle-sized species such as $M$. costaricensis $(8.5-10 \mathrm{~mm})$, and very small species such as T. pipioli $(2.5-3 \mathrm{~mm})$. These differences are also reflected by their brain sizes, as Figure $7 \mathrm{~b}$ indicates. Here, a comparative morphometric brain analysis must show whether this is a general finding within the corbiculate bees. For several other (eusocial) insects, such as ants and bumblebees, it was shown that smaller animals have smaller brains [Mares et al., 2005; Wehner et al., 2007]. However, Riveros and Gronenberg [2009] 
showed a correlation between body size and learning performance. It was shown that tyramine can influence gustatory responsiveness [Scheiner et al., 2002; Scheiner et al., 2017a] and thus learning performance. It is therefore an intriguing question whether brain miniaturization has an effect on the spatial expression of the respective receptors. We did not find qualitative differences in brain TAR1-IR of the species analyzed here, which could be associated with miniaturization. This additionally supports our interpretation of a highly conserved TAR1 expression in the brain and stresses the importance of this receptor for behavioral regulation. This is possibly true for all monoaminergic neuromodulators and their receptors, as suggested by results from the parasitic wasp Trichogramma evanescens, which is an extreme example of a miniaturized insect with an adult body length of only $0.3 \mathrm{~mm}$. Interestingly, the brain of this small insect has the same set of monoaminergic neurons as A. mellifera, even though dopaminergic and some octopaminergic neuron clusters contain fewer neurons [van der Woude and Smid, 2017].

\section{Conclusion and Outlook}

Our results indicate that the tyramine 1 receptor possesses functions in the processing, the modulation, and the integration of sensory information in insects. Furthermore, most of these functions seem to be conserved as the spatial distribution of the receptor in the different bee species suggests. However, our results additionally show that expression data gained in a well-established model organism cannot be transferred one-to-one to closely related species, as the results of the optic lobes indicates. Furthermore, this expression flexibility in the optic lobes indicates that it might be worthwhile to explore it in more detail in the honeybee, as it may indicate the presence of expression-influencing factors that we do not understand. Finally, we do not know whether similarities in TAR1 brain expression patterns indeed mean that this receptor has identical functions across species even if they are closely related. Although this explanation seems logi$\mathrm{cal}$, future comparative studies must show whether this is actually the case.

\section{Statement of Ethics}

An ethics statement is not applicable, as there are no review boards for work on insects.

\section{Disclosure Statement}

The authors declare that they have no conflicts of interest.

\section{Funding Sources}

There are no funding sources to declare.

\section{Author Contributions}

M.T., R.S., and A.B.: conceptualization and methodology. M.T. and K.W.: investigation and acquisition of the data. M.T., K.W., and R.S.: data analysis. M.T., R.S., and A.B.: writing.

\section{References}

Barron AB, Gurney KN, Meah LF, Vasilaki E, Marshall JA. Decision-making and action selection in insects: inspiration from vertebratebased theories. Front Behav Neurosci. 2015 Aug;9:216.

Barron AB, Robinson GE. Selective modulation of task performance by octopamine in honey bee (Apis mellifera) division of labour. J Comp Physiol A Neuroethol Sens Neural Behav Physiol. 2005 Jul;191(7):659-68.

Barron AB, Schulz DJ, Robinson GE. Octopamine modulates responsiveness to foraging-related stimuli in honey bees (Apis mellifera). J Comp Physiol A Neuroethol Sens Neural Behav Physiol. 2002 Sep;188(8):603-10.
Blenau W, Balfanz S, Baumann A. Amtyr1: characterization of a gene from honeybee (Apis mellifera) brain encoding a functional tyramine receptor. J Neurochem. 2000 Mar;74(3):900-8.

Branstetter MG, Danforth BN, Pitts JP, Faircloth BC, Ward PS, Buffington ML, et al. Phylogenomic insights into the evolution of stinging wasps and the origins of ants and bees. Curr Biol. 2017 Apr;27(7):1019-25.

Brockmann A, Robinson GE. Central projections of sensory systems involved in honey bee dance language communication. Brain Behav Evol. 2007;70(2):125-36.

Damrau C, Toshima N, Tanimura T, Brembs B, Colomb J. Octopamine and tyramine contribute separately to the counter-regulatory response to sugar deficit in Drosophila. Front Syst Neurosci. 2018 Jan;11:100.
Değirmenci L, Geiger D, Rogé Ferreira FL, Keller A, Krischke B, Beye M, et al. CRISPR/Cas 9-Mediated Mutations as a New Tool for Studying Taste in Honeybees. Chem Senses. 2020 Nov;45(8):655-66.

Değirmenci L, Thamm M, Scheiner R. Responses to sugar and sugar receptor gene expression in different social roles of the honeybee (Apis mellifera). J Insect Physiol. 2018 Apr;106(Pt 1):65-70.

Ehmer B, Gronenberg W. Segregation of visual input to the mushroom bodies in the honeybee (Apis mellifera). J Comp Neurol. 2002 Sep;451(4):362-73.

El-Kholy S, Stephano F, Li Y, Bhandari A, Fink C, Roeder T. Expression analysis of octopamine and tyramine receptors in Drosophila. Cell Tissue Res. 2015 Sep;361(3):669-84. 
Finetti L, Roeder T, Calò G, Bernacchia G. The Insect Type 1 Tyramine Receptors: From Structure to Behavior. Insects. 2021 Apr; 12(4):315

Fussnecker BL, Smith BH, Mustard JA. Octopamine and tyramine influence the behavioral profile of locomotor activity in the honey bee (Apis mellifera). J Insect Physiol. 2006 Oct; 52(10):1083-92.

Gonzalez VH, Griswold T, Praz CJ, Danforth BN. Phylogeny of the bee family Megachilidae (Hymenoptera: Apoidea) based on adult morphology. Syst Entomol. 2012;37(2):26186.

Guo X, Wang Y, Sinakevitch I, Lei H, Smith BH. Comparison of RNAi knockdown effect of tyramine receptor 1 induced by dsRNA and siRNA in brains of the honey bee, Apis mellifera. J Insect Physiol. 2018 Nov - Dec;111:47-52.

Haupt SS. Central gustatory projections and sidespecificity of operant antennal muscle conditioning in the honeybee. J Comp Physiol A Neuroethol Sens Neural Behav Physiol. 2007 May;193(5):523-35.

Held M, Berz A, Hensgen R, Muenz TS, Scholl C, Rössler W, et al. Microglomerular Synaptic Complexes in the Sky-Compass Network of the Honeybee Connect Parallel Pathways from the Anterior Optic Tubercle to the Central Complex. Front Behav Neurosci. 2016 Oct; $10: 186$.

Homberg U. In search of the sky compass in the insect brain. Naturwissenschaften. 2004 May; 91(5):199-208.

Huang ZY, Robinson GE. Honeybee colony integration: worker-worker interactions mediate hormonally regulated plasticity in division of labor. Proc Natl Acad Sci USA. 1992 Dec; 89(24):11726-9.

Hunt GJ, Amdam GV, Schlipalius D, Emore C, Sardesai N, Williams CE, et al. Behavioral genomics of honeybee foraging and nest defense. Naturwissenschaften. 2007 Apr;94(4): 247-67.

Jain R, Brockmann A. Sex-specific molecular specialization and activity rhythm-dependent gene expression in honey bee antennae. J Exp Biol. 2020 Jun;223(Pt 12):jeb217406.

Kawakita A, Ascher JS, Sota T, Kato M, Roubik DW. Phylogenetic analysis of the corbiculate bee tribes based on 12 nuclear protein-coding genes (Hymenoptera: Apoidea: Apidae). Apidologie. 2008;39(1):163-75.

Kenyon FC. The brain of the bee. A preliminary contribution to the morphology of the nervous system of the arthropoda. J Comp Neurol. 1896;6(3):133-210.

Kirschner S, Kleineidam CJ, Zube C, Rybak J, Grünewald B, Rössler W. Dual olfactory pathway in the honeybee, Apis mellifera. J Comp Neurol. 2006 Dec;499(6):933-52.

Kutsukake M, Komatsu A, Yamamoto D, IshiwaChigusa S. A tyramine receptor gene mutation causes a defective olfactory behavior in Drosophila melanogaster. Gene. 2000 Mar; 245(1):31-42.
Mares S, Ash L, Gronenberg W. Brain allometry in bumblebee and honey bee workers. Brain Behav Evol. 2005;66(1):50-61.

McCabe SI, Ferro MW, Farina WM, Hrncir M. Dose-and time-dependent effects of oral octopamine treatments on the sucrose responsiveness in stingless bees (Melipona scutellaris). Apidologie. 2017;48(1):1-7.

Mustard JA, Kurshan PT, Hamilton IS, Blenau W, Mercer AR. Developmental expression of a tyramine receptor gene in the brain of the honey bee, Apis mellifera. J Comp Neurol. 2005 Feb;483(1):66-75.

Nisimura T, Seto A, Nakamura K, Miyama M, Nagao T, Tamotsu S, et al. Experiential effects of appetitive and nonappetitive odors on feeding behavior in the blowfly, Phormia regina: a putative role for tyramine in appetite regulation. J Neurosci.2005 Aug;25(33):750716.

Patwardhan A, Cheng N, Trejo J. Post-Translational Modifications of $\mathrm{G}$ Protein-Coupled Receptors Control Cellular Signaling Dynamics in Space and Time. Pharmacol Rev. 2021 Jan;73(1):120-51.

Peng T, Schroeder M, Grüter C. Octopamine increases individual and collective foraging in a neotropical stingless bee. Biol Lett. 2020 Jun;16(6):20200238.

Pfeiffer K, Homberg U. Organization and functional roles of the central complex in the insect brain. Annu Rev Entomol. 2014;59(1): $165-84$.

Reim T, Balfanz S, Baumann A, Blenau W, Thamm M, Scheiner R. AmTAR2: functional characterization of a honeybee tyramine receptor stimulating adenylyl cyclase activity. Insect Biochem Mol Biol. 2017 Jan;80:91-100.

Riveros AJ, Gronenberg W. Learning from learning and memory in bumblebees. Commun Integr Biol. 2009 Sep;2(5):437-40.

Robinson GE. Regulation of division of labor in insect societies. Annu Rev Entomol. 1992, 37(1):637-65

Rodriguez-Serrano E, Inostroza-Michael O, Avaria-Llautureo J, Hernandez CE. Colony size evolution and the origin of eusociality in corbiculate bees (Hymenoptera: apinae). PLoS One. 2012;7(7):e40838.

Rotte C, Krach C, Balfanz S, Baumann A, Walz B, Blenau W. Molecular characterization and localization of the first tyramine receptor of the American cockroach (Periplaneta americana). Neuroscience. 2009 Sep;162(4):112033.

Rybak J, Menzel R. Mushroom body of the honeybee. In: Shepherd GM, Grillner S, editors. Handbook of brain microcircuits. Oxford, Oxford University Press; 2010. pp. 433-438.

Sandoz JC. Olfaction in honey bees: from molecules to behavior. In: Galizia CG, Eisenhardt D, Giurfa M, editors. Honeybee neurobiology and behavior. Springer Netherlands; 2012. https://doi.org/10.1007/978-94-007-20992_19.
Sann M, Niehuis O, Peters RS, Mayer C, Kozlov A, Podsiadlowski L, et al. Phylogenomic analysis of Apoidea sheds new light on the sister group of bees. BMC Evol Biol. 2018 May;18(1):71.

Saraswati S, Fox LE, Soll DR, Wu CF. Tyramine and octopamine have opposite effects on the locomotion of Drosophila larvae. J Neurobiol. 2004 Mar;58(4):425-41.

Scheiner R, Entler BV, Barron AB, Scholl C, Thamm M. The Effects of Fat Body Tyramine Level on Gustatory Responsiveness of Honeybees (Apis mellifera) Differ between Behavioral Castes. Front Syst Neurosci. 2017b Aug;11:55.

Scheiner R, Page RE Jr, Erber J. The effects of genotype, foraging role, and sucrose responsiveness on the tactile learning performance of honey bees (Apis mellifera L.). Neurobiol Learn Mem. 2001 Sep;76(2):138-50.

Scheiner R, Plückhahn S, Oney B, Blenau W, Erber J. Behavioural pharmacology of octopamine, tyramine and dopamine in honey bees. Behav Brain Res. 2002 Nov;136(2):545-53.

Scheiner R, Reim T, Søvik E, Entler BV, Barron $A B$, Thamm M. Learning, gustatory responsiveness and tyramine differences across nurse and forager honeybees. J Exp Biol. 2017a Apr;220(Pt 8):1443-50.

Sinakevitch IT, Daskalova SM, Smith BH. The Biogenic Amine Tyramine and its Receptor (AmTyr1) in Olfactory Neuropils in the Honey Bee (Apis mellifera) Brain. Front Syst Neurosci. 2017 Oct;11:77.

Strausfeld NJ. Organization of the honey bee mushroom body: representation of the calyx within the vertical and gamma lobes. J Comp Neurol. 2002 Aug;450(1):4-33.

Strauss R. The central complex and the genetic dissection of locomotor behaviour. Curr Opin Neurobiol. 2002 Dec;12(6):633-8.

Thamm M, Scheiner R. PKG in honey bees: spatial expression, Amfor gene expression, sucrose responsiveness, and division of labor. J Comp Neurol. 2014 Jun;522(8):1786-99.

Thamm M, Scholl C, Reim T, Grübel K, Möller K, Rössler W, et al. Neuronal distribution of tyramine and the tyramine receptor AmTAR1 in the honeybee brain. J Comp Neurol. 2017 Aug;525(12):2615-31.

Thamm M, Sturm K, Schlossmann J, Scheiner R. Levels and activity of cyclic guanosine monophosphate-dependent protein kinase in nurse and forager honeybees. Insect Mol Biol. 2018 Dec;27(6):815-23.

van der Woude E, Smid HM. Maximized complexity in miniaturized brains: morphology and distribution of octopaminergic, dopaminergic and serotonergic neurons in the parasitic wasp, Trichogramma evanescens. Cell Tissue Res. 2017 Sep;369(3):477-96.

Vogel C, Marcotte EM. Insights into the regulation of protein abundance from proteomic and transcriptomic analyses. Nat Rev Genet. 2012 Mar;13(4):227-32.

Wehner R, Fukushi T, Isler K. On being small: brain allometry in ants. Brain Behav Evol. 2007;69(3):220-8.
TAR1 Brain Distribution in Corbiculate Bees
Brain Behav Evol 2021;96:13-25 DOI: 10.1159/000517014 